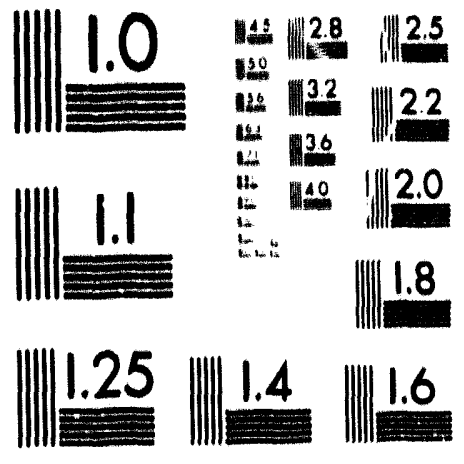



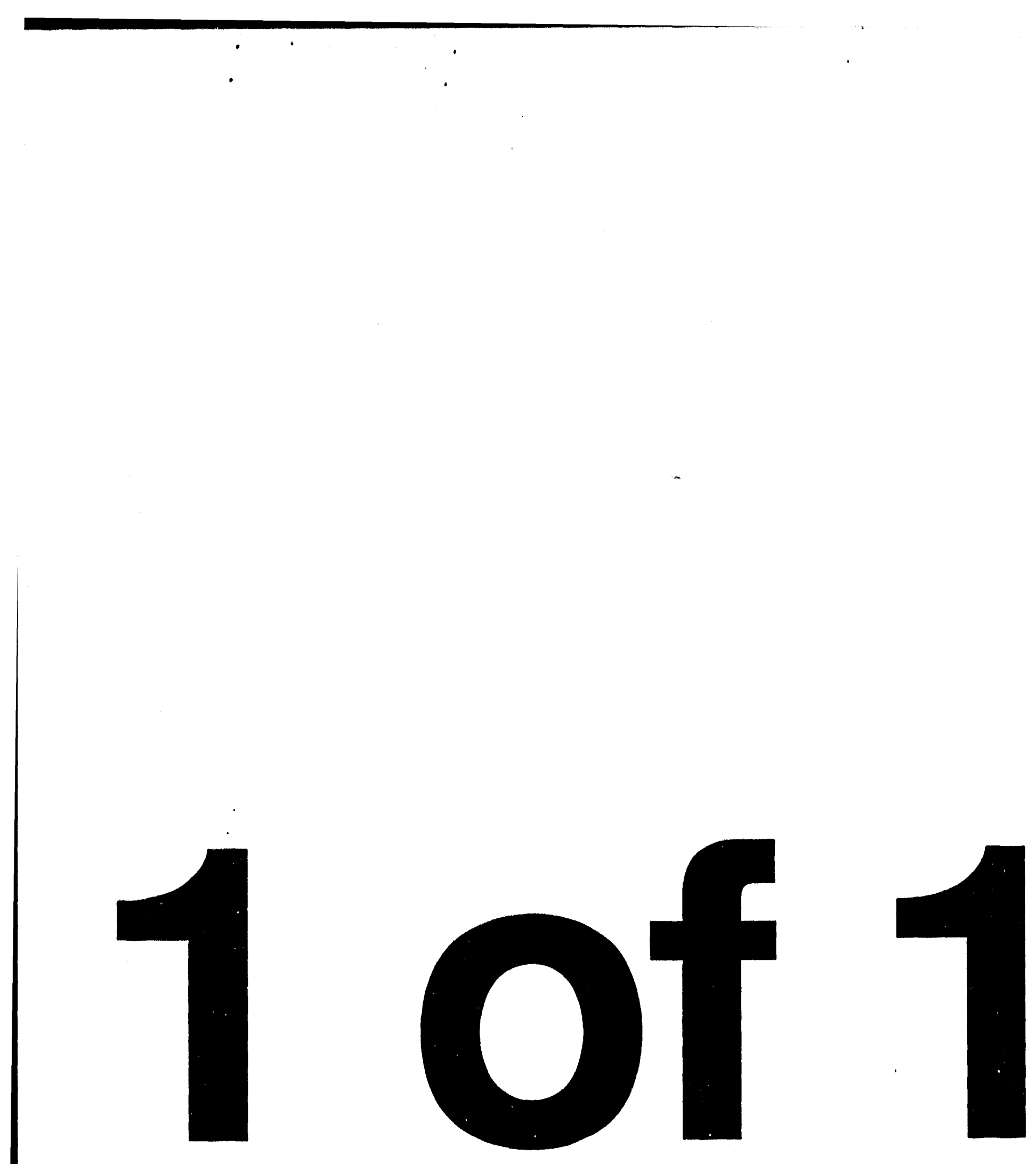
GEM TN.92-182

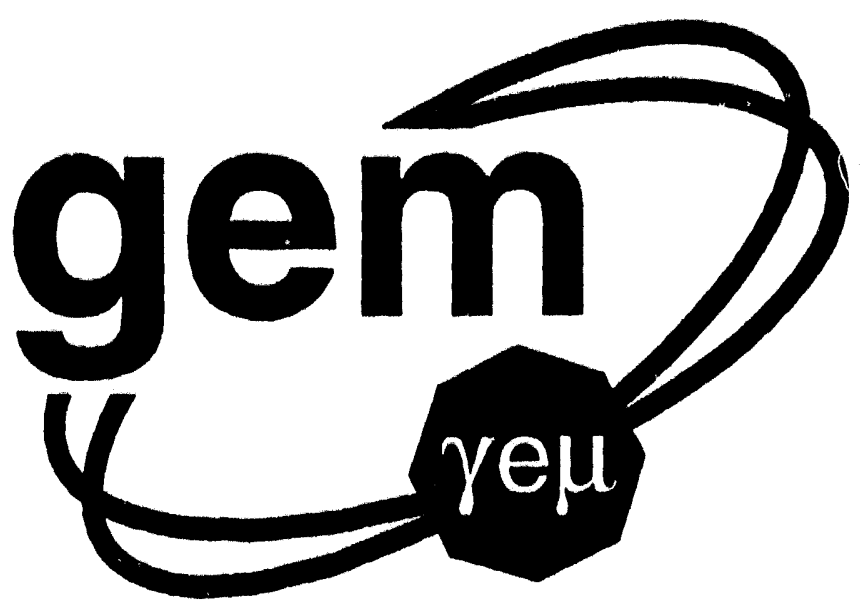

\section{Effects of Light Exposure on Irradiated Barium Fluoride Crystals}

Craig R. Wuest, G. Joseph Mauger

Lawrence Livermore National Laboratory

August 27, 1992

Abstract:

Small barium fluoride crystals have been irradiated using Cobalt -60 gamma rays under various illumination conditions to establish the effect of photo-bleaching of the radiation-induced color centers. This paper describes results of a few different experiments conducted at LLNL over the past few weeks. 


\title{
Effects of Light Exposure on Irradiated Barium Fluoride Crystals
}

\author{
Craig R. Wuest, G. Joseph Mauger \\ Lawrence Livermore National Laboratory
}

August 27, 1992

\begin{abstract}
Small barium fluoride crystals have been irradiated using Cobalt-60 gamma rays under various illumination conditions to establish the effect of photobleaching of the radiation-induced color centers. This paper describes results of a few different experiments conducted at LLNL over the past few weeks.
\end{abstract}

\section{Introduction}

At the recommendation of the Barium Fluoride Review Panel ${ }^{1}$, a study of optical annealing of irradiated barium fluoride crystals was begun at LLNL. The purpose of the study was to first verify that optical annealing is feasible and then to perform in-situ annealing during irradiation to determine the effect on the optical properties of the crystal.

Two small barium fluoride cubes with dimensions 1 inch on each side were used in this study. These crystals were recently brought back from the Shanghai Institute of Ceramics (SIC) and are presumably from the more recent, high quality crystals grown at SIC. Both crystals were polished using the standard LLNL diamond-loaded pitch polishing. One crystal had all six sides polished, whereas the other crystal had only two opposite faces polished. The remaining faces of this crystal were rough ground. No annealing of the crystals was performed prior to the first set of measurements.

All measurements were made using a Perkin Elmer UV/VIS/NIR spectrophotometer. This spectrophotometer is configured with the monochromator before the sample so that the sample only is illuminated with light of a particular color. The manufacturer claims measurement accuracy of about $0.07 \%$ and repeatability of $0.03 \%$. Care was taken to keep the samples in the dark except when illumination was required for annealing purposes.

\section{Experimental Results}

\section{A. Irradiation in-situ with UV light:}

The first experiment irradiated the crystal with all 6 sides polished. This crystal was placed in a dry well in the cobalt 60 source tank and it received $84 \mathrm{krad}$ total exposure over a 29 hour period, corresponding to a dose rate of about $2.9 \mathrm{krad} /$ hour. The crystal was exposed to a weak mercury lamp (principal line $-254 \mathrm{~nm}$ ) placed 1 inch from one of 
the faces durine gamma irradiation. The lamp is a UVP, Inc. Pen-Ray laboratory light source with a rating of 0.004 watts $/ \mathrm{cm}^{2}$ at 1 inch distance. Figure 1 shows three curves: a) the initial transmission through the crystal versus wavelength, b) the transmission after irradiation while being illuminated and c) the transmission after 4 days storage in a glass dessicator, while exposed to normal fluorescent room lights at room temperature.

At $220 \mathrm{~nm}$ the transmission is seen to decrease from $88.9 \%$ to $87.5 \%$ and then to increase to $88.5 \%$ after 4 days of storage in ambient room light. From reference (2) this corresponds to attenuation lengths of $201 \mathrm{~cm}, 89 \mathrm{~cm}$, and $148 \mathrm{~cm}$, respectively.

\section{B. Irradiation in darkness followed by optical annealing:}

This experiment used the crystal with the two opposite faces polished. The crystal had been irradiated previously and was annealed in a vacuum oven at $500 \mathrm{C}$ for 2 hours. However the initial transmission of this crystal is not as good as the crystal in Experiment A (initial transmission $=86.75 \%$ ). It is thought that the surface may have been contaminated during the initial annealing step. To verify this the crystal was analyzed using a scanning electron microscope sensitive to elements on the surface and down to about 0.5 $\mu \mathrm{m}$ depth into the crystal. The results indicate small amounts of tungsten and larger amounts of chlorine are present on the surface of the crystal, presumably due to contamination from the vacuum oven. However for the purposes of this experiment we will examine the relative effects of irradiation and optical annealing based on the initial conditions measured.

The crystal was irradiated in darkness to a total dose of $84 \mathrm{krad}$ at $2.9 \mathrm{krad} / \mathrm{hour}$. Following irradiation the crystal's transtnission was measured and then the crystal was exposed to a white light source ( 20 watt tungsten lamp) through a 0.25 inch diameter 1 meter long silica fiber optic bundle. Two measurements of transmission were made - one at 30 minutes total exposure to the light and one at 17 hours total exposure. The crystal was then exposed for an additional 1.25 hours to the UV lamp described in experiment $A$.

Figure 2 shows 5 curves for this experiment. Curve a) gives the initial transmission through the crystal. Curve b) gives the transmission after irradiation (the crystal was kept in darkness during this irradiation). Curve c) gives the transmission after 30 minutes exposure to white light. Curve d) gives the transmission after 17 minutes exposure to white light. Curve e) gives the transmission after 1.25 hours exposure to UV light.

At $220 \mathrm{~nm}$ the transmission is seen to drop from $86.75 \%$ to $79.63 \%$. After 30 minutes exposure to white light the transmission increases to $84 \%$ and after an additional 16.5 hours of white light exposure the transmission increases to $85.25 \%$. An additional 1.25 hours of UV exposure increases the transmission to $88 \%$, which is greater than the initial transmission. This could be due to changes in the surface (drying) during illumination or the removal of some surface contamination by the UV photons.

\section{Discussion}

We can establish the general effects of optical annealing from these experiments. First, optical annealing in-situ has the effect of reducing the relative amount of radiationinduced darkening of the crystal compared to a crystal irradiated in darkness. For the conditions of this experiment the in-situ illuminated crystal had its transmission reduced 
by $1.4 \%$ compared to $7.1 \%$ reduction for the unilluminated crystal under the same irradiation conditions. This corresponds to a factor of 5 reduction in the induced darkening.

Another effect, also seen in experiments at West Virginia University $(\mathrm{WVU})^{3}$ is a rapid bleaching of the crystal upon illumination by light for a few tens of minutes followed by a much reduced bleaching rate. This can be seen by comparing curves $b$ ), $c$ ) and d) in Figure 2.

Finally, it appears that the addition of shorter wavelength UV light after exposure to longer wavelength light can further bleach a crystal that had approached saturation at the longer wavelengths. This effect has been observed at WVU and at CalTech.

We note that the optical power used in these tests to achieve measurable bleaching is small, on the order of milliwatts $/ \mathrm{cm}^{2}$ for white and UV light. In discussions with experts on power handling characteristics of fiber optics, we have learned that standard silica fibers similar to those proposed for the Barium Fluoride Calorimeter can carry substantial amounts of optical power. For example LLNL's Laser Isotope Separation Program (LIS) uses copper vapor lasers for pumping dyes to produce laser light of specific frequencies for atomic ionization and isotopic separation of atomic vapors. The LIS Program is actively researching the use of silica (glass core and glass cladding) fibers of $0.5 \mathrm{~mm}$ diameter for transporting the $511 \mathrm{~nm}$ copper vapor laser light. They have identified a $3 \mathrm{M}$ fiber that is capable of handling 200 watts time-averaged power (the laser is pulsed at $100 \mathrm{~Hz}$ ). As much as 800 watts of optical power has been transported in $1 \mathrm{~mm}$ core silica fibers. Discussions with experts in LIS have indicated that a $100 \mu \mathrm{m}$ diameter multi-mode fiber can deliver on the order of watts without deterioration. LLNL will obtain samples of the $0.5 \mathrm{~mm}$ fiber for additional experiments on optical bleaching of crystals in-situ under more realistic dose rate conditions.

\section{References:}

1. "Report of the $\mathrm{BaF}_{2}$ Panel after its third meeting on August 3-4, 1992," unpublished, August 5, 1992.

2. Da-an Ma and Ren-yuan Zhu, "Light Attenuation Length of Barium Fluoride Crystals," CALT-68-1811, GEM-TN-92-148, August 30, 1992.

3. Larry Halliburton, et al., "Effect of Optical Bleaching on Radiation-induced Defects in $\mathrm{BaF}_{2}$," West Virginia University Technical Report, August 22, 1992. 
Figure 1

SAMPLE EAF2-12, Exp series 2

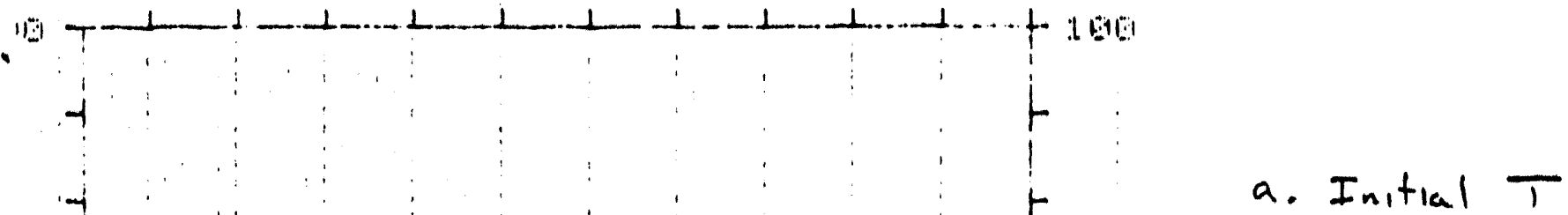

b. After coco exposure

$2.9 \frac{\mathrm{kmed}}{\mathrm{hr}} \times 29 \mathrm{hrs}=84 \mathrm{krad}$ with UV', illumination $254 \mathrm{~nm} Q .004 \mathrm{w} / \mathrm{cm}^{2}$

6. After 4 days storage in dessicator w/ room lights.

: $19-1$

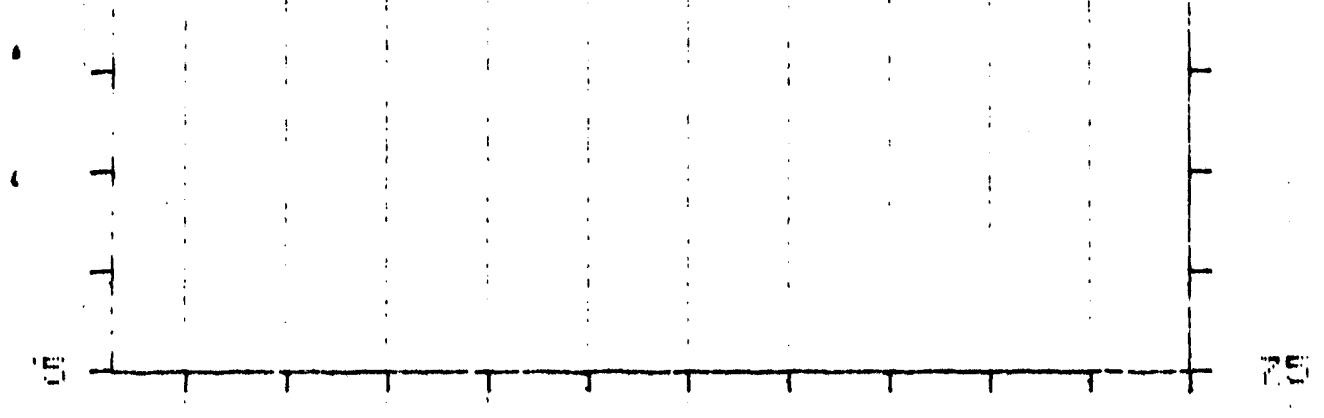

OI: $B A F Z-12$

3

405 

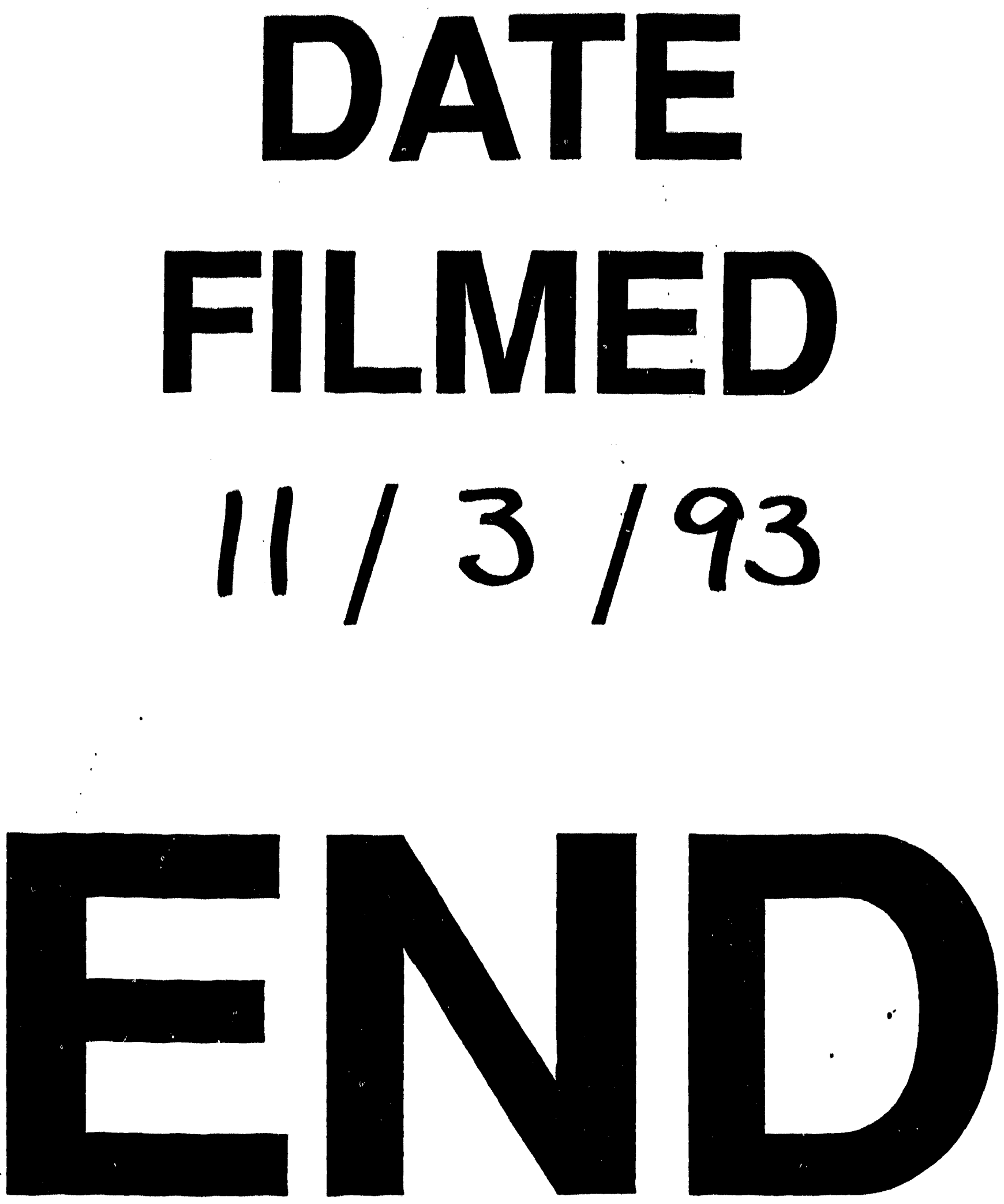
